\title{
SUJETO Y SUBJETIVIDAD: UNA APROXIMACIÓN DESDE LAS REPRESENTACIONES ESPACIALES
}

\author{
SUBJECT AND SUBJECTIVITY: AN APPROACH FROM SPACE REPRESENTATIONS
}

Iliana Araya Ramírez

Universidad Nacional (UNA), Heredia, Costa Rica, ilianaraya@gmail.com

\section{RESUMEN}

El objetivo central de este ensayo consiste en comprender las representaciones espaciales desde la mirada del sujeto y la subjetividad. El "giro espacial" abre las posibilidades de interpretación del espacio a partir una perspectiva interdisciplinaria donde la centralidad del sujeto y la subjetividad recobran el interés por parte de la Geografía. La articulación entre la dimensión material y sensible del espacio conlleva la construcción social; de ahí el interés por el lugar y el simbolismo en su dimensión sensible, ya que las formas de representación espacial se concretan en los lugares. De esta forma, la "trialéctica de la espacialidad" abre el diálogo interdisciplinario desde la subalternidad como una forma de resistencia a las representaciones hegemónicas en el contexto de la globalización.

Palabras claves: Sujeto y subjetividad; trialéctica de la espacialidad; lugar; representaciones espaciales; diálogo interdisciplinario.

\section{ABSTRACT}

The main objective of this paper is to understand spatial representations from the perspective of the subject and subjectivity. The "spatial turn" opens the possibilities of interpretation of space from an interdisciplinary perspective where the centrality of the subject and subjectivity regain the interest of Geography. The relationship between the material and sensible space dimension involves the social construction; hence the interest in the place and symbolism in his sensitive dimension, as forms of spatial representation are specified on places. Thus, the "trialectics of spatiality" open interdisciplinary dialogue from subalternity as a form of resistance to hegemonic representations in the context of globalization.

Keywords: Subject and subjectivity; trialectics of spatiality; place; spatial representations; interdisciplinary dialogue.

Artigo recebido para publicação em agosto de 2015

Artigo aceito para publicação em outubro de 2015

\section{INTRODUÇÃO}

En la actualidad, los cambios que ocurren en la geografía humana, señalados como giros, abren la posibilidad de diálogos entre el orden de lo social con lo cultural, lo cual contribuye a los aportes de la investigación en ciencias sociales. Inclusive, en la concepción de lo social, en términos de "sujetos sociales territorializados, entendidos como agentes activos que pueden transformar la sociedad" (Lindón, 2012, p. 588).

El interés acerca de la espacialidad, por parte de otras distintas disciplinas, forma parte del denominado "giro espacial", el cual consiste en el reconocimiento e interpretación de la espacialidad de 
la vida humana, con un poder interpretativo concedido tradicionalmente al tiempo y a las relaciones sociales (Benach \& Albert, 2010). Así cuando autores de las diferentes áreas estudian la cultura, idioma, religión, género, clase y representaciones sociales reconocen el sentido de lugar con relación a lo social y ambiental. De esta manera, surgen los diálogos interdisciplinarios y transdisciplinarios como aproximación a algunos conceptos claves, que desde siempre fueron entendidos por la geografía. Estos eruditos pueden ser lingüistas, novelistas, críticos de cine, antropólogos, sociólogos, historiadores y politólogos.

Cuando en la construcción epistemológica de la teoría social se invisibiliza de la dimensión espacial de lo social, se está cercenando lo social que se quiere comprender, aunque muchos científicos sociales comprometidos con la coordenada espacial de lo social omiten el devenir geográfico (Lindón, 2012b).

La representación única del espacio se impuso como una verdad absoluta sobrepuesta sobre otras formas (Boynard, 2013). Por esta razón, la hegemonía del concepto espacio, sobre el de lugar, coexiste como una forma de colonialismo occidental y actúa como un mecanismo epistemológico del eurocentrismo en la construcción de la teoría social. Para Escobar "Al restarle énfasis a la construcción cultural del lugar al servicio del proceso abstracto y aparentemente universal de la formación del capital y del Estado, casi toda la teoría social convencional ha hecho invisibles formas subalternas de pensar y modalidades locales y regionales de configurar el mundo" (2000, p. 116).

En la actualidad, se presentan otras formas alternativas para el abordaje de la realidad social y las representaciones del mundo, por esta razón cada vez es más frecuente la incorporación del sujeto y la subjetividad como derroteros de la investigación en las ciencias sociales (Pérez, 2013). El devenir de la subjetividad en la geografía refiere a una ruptura con la visión cartesiana y euclidiana del espacio. De hecho, desde los años setenta se utiliza en la Geografía Humana, la concepción del espacio como experiencia, la cual se impulsó, en las figuras de Yi Fu Tuan (1977) y Anne Buttimer (1980) (Citado en Lindón, 2012b). 
De ahí, la distinción entre los conceptos de espacio y lugar, para Tuan (1977) el lugar hace referencia a aquellos espacios delimitados que presentan certeza y seguridad para los sujetos (Lindón, Aguilar \& Hiernaux, 2006). Los lugares adquieren un sentido simbólico por medio de la objetivación de los elementos, los cuales son codificados en redes de comunicación y las relaciones con el entorno (Lindón et al, 2006). En su libro Topofilia, Tuan aborda la dimensión sensible del espacio en cuanto a lo sensorial, perceptual y como experiencia de la persona; este autor reconstruye el concepto de topofilia, en el cual los sujetos otorgan subjetividad al espacio percibido, apropiado, habitado y significado. El espacio como experiencia se circunscribe al concepto de lugar, donde este último se considera un territorio de significaciones (Tuan, 2006). Esta perspectiva concuerda con (Augé, 2008) la cual indica que el lugar está cargado de significados, en donde las prácticas socioespaciales les otorgan significado.

La concepción de experiencia y vivencia espacial aproxima a la construcción social de los lugares entre la materialidad y la subjetividad, lo que Claude Raffestin denomina "semiotización del espacio" al incorporar los signos culturales del espacio material característico de una sociedad (Raffestin, 1986. Citado en Lindón, 2012). Esta concepción resulta afín al momento actual dada la presencia del giro cultural y la centralidad del sujeto en relación con otras disciplinas (Lindón, 2007).

Por tanto, se retoma el interés por otras formas de representación del espacio, distintas a la abstracta, que aluden, inevitablemente, a la incorporación de la dimensión sensible del espacio. De ahí que comprender las representaciones espaciales desde la mirada del sujeto y la subjetividad constituye el objetivo central de este ensayo.

\section{Sujeto, subjetividad y su relación con el espacio}

El sujeto moderno remite al individualismo y el hedonismo, de forma que la restricción al cuerpo busca el bienestar del mismo, por medio del placer inmediato (Pérez, 2009). Por el contrario, para Pozzoli el sujeto complejo “...se introduce progresivamente en un proceso dialógico y reflexivo, que implica la expansión y reorganización de la conciencia" (2006, p. 5). Es quizás este nivel de conciencia el que 
puede conducir a una sociedad con mayor capacidad emancipadora, porque un nivel de conciencia distinto, al prevaleciente en una sociedad individualista, trasciende a la persona. Por consiguiente, en el ejercicio de la reflexividad, el sujeto despliega estrategias metacognitivas y de forma concomitante “...comienza a auto-observarse ejerciendo su capacidad de sujeto histórico, lo que le permite recuperar su protagonismo y orientarse de un modo más autónomo" (p. 5) El ejercicio de reflexividad plantea una nueva forma de re-existir y ser un sujeto agente de cambio ante las múltiples miradas.

En esta misma dirección, Najmanovich (2011) señala que en la sociedad actual requiere de otros escenarios que incorporen la dimensión subjetiva y propicien un acercamiento a lo multidimensional. Para esto son necesarias otras "formas de acceder el espacio cognitivo-experencial caracterizados por las formaciones de bucles" (p. 93). Esta posición coincide con Ciurana (1997) donde la subjetividad del sujeto produce retroalimentación de forma recursiva y dinámica a manera de bucles, en relación con otro, que a su vez permite el descubrimiento de mi diversidad y puede ser un punto de encuentro (Citado en Pérez, 2013).

Desde una perspectiva espacial, esto implica un devenir de la subjetividad y de un sujeto social que construye al objeto de manera indisociable y solidaria; es decir, el sujeto social, interactúa en el espacio y simultáneamente este es transformado en su interacción con él. Para Najmanovich (2011, p.93) "no nacemos "sujetos" sino que devenimos tales en y a través del juego social". De igual manera devenimos en sujetos sociales por medio de la interacción con nuestro espacio, y de manera específica en los lugares. Tal y como lo señala Escobar (2000) "las mentes se despiertan en un mundo, pero también en lugares concretos, y el conocimiento local es un modo de conciencia basado en el lugar, una manera lugar-específica de otorgarle sentido al mundo" (p.125).

La centralidad del sujeto y la subjetividad en las Ciencias Sociales está presente desde las últimas tres décadas (Lindón, 2009) y forman parte de las reflexiones de la psicología, la sociología y la antropología y, más recientemente de la economía, la historia, el derecho y las ciencias políticas (Pérez, 2013). En tanto los puentes entre la Teoría Social y la Geografía Humana que integran el cuerpo y las emociones son más recientes. La relación entre el sujeto y el espacio proviene de los años setenta, 
en las figuras de Giddens (desde la Sociología) y Hägerstrand (desde la Geografía Humana), tal y como afirma Lindón (2009) "En buena medida la fecundidad de este debate radica en que terminó abriendo el camino para pensar la "reproducción /producción" en términos socioespaciales y no exclusivamente sociales" (p. 9).

Desde esta perspectiva, la relación entre sujeto y espacio otorga centralidad al sujeto desde dos formas distintas, por un lado el sujeto-habitante que construye lo social, y lo urbano en particular; y por otro el sujeto-habitante en el sujeto-cuerpo y el sujeto-sentimiento (Lindón, 2009). Así, la mirada del "sujeto habitante, con su corporeidad y emocionalidad, resultan fecundas para comprender las ciudades porque lo urbano lleva consigo una dimensión espacial insoslayable, tanto en lo que respecta a las formas espaciales (lo morfológico) como en cuanto a la espacialidad de la experiencia urbana, o la espacialidad del habitar la ciudad" (Lindón, 2009, p.11).

Para Pérez (2013) la relación entre el sujeto y la subjetividad se escinde, de forma que la subjetividad es una configuración y el sujeto es un agente de cambio. La subjetividad está entendida “...como la dialéctica entre sincronía y diacronía de los procesos de construcción que hace posible, en determinado momento del desarrollo histórico, dar cuenta de cierta configuración de representaciones, afectos y disposiciones volitivas que caracterizan determinados grupos sociales" (p. 21). El sujeto-habitante como agente de cambio, en la construcción socio-espacial de la ciudad, configura las representaciones espaciales de los grupos que habitan el espacio urbano.

La configuración de tales representaciones incorporan la dimensión sensible del espacio, la cual alude a la experiencia geográfica que afecta nuestra manera de actuar en el espacio, así como la relación con los otros, en la producción de un tejido social (Hiernuaux-Nicolas, 2008). Según, Debarbieux (1997) el geógrafo busca comprender como se percibe y configura la experiencia y la forma en que esta contribuye en la construcción del espacio (Citado en Hiernaux, 2008).

\section{La trialéctica espacial: de las representaciones hegemónicas a las contra- representaciones del espacio}


En este ensayo, el estudio de lo local y el lugar se aborda desde lo que Soja (1996) denomina la "trialéctica de la espacialidad"; basado en los planteamientos de Lefevre (1996) en su libro La production de l! espace; este autor plantea la alternativa del tercer espacio para superar el dualismo restrictivo entre el espacio percibido y el espacio concebido (Benach \& Albert, 2010). Soja (1996) en su libro Thirdspace: Journeys to Los Angeles and Others Real-and-Imagined Places elabora una interpretación del espacio donde interactúan los elementos materiales y simbólicos, además completa la idea de la diferencia donde confluyen múltiples escalas (Clua \& Zusman, 2002). Soja plantea la tríada ontológica; el primer espacio (espacio percibido) se refiere a aquel que representa fenómenos cartografiables y es empíricamente sometido a la medición, se interesa en "descripciones precisas de patrones y distribuciones (como el estudio de diferenciación de áreas), la búsqueda de regulaciones empíricas (...), la correlación de la covarianza espacial de una configuración geográfica con otra" (Benach \& Albert, 2010, p. 188-189). El segundo espacio (espacio concebido) se preocupa por "las imágenes y las representaciones de la espacialidad” y (...) "explora los mundos más cognitivos, conceptuales y simbólicos" (Bebach \& Albet, 2010, p. 189). En cambio, el tercer espacio (vivido), en tanto real como simbólico, trasciende el dualismo entre el primero y el segundo; lo que estimula la imaginación geográfica e invita a la diferencia y la otredad "como una manera radicalmente diferente de ver, interpretar, y actuar para cambiar la espacialidad que abarca toda la vida humana" por medio de los espacios de representación (Bebach \& Albet, 2010, p.191). Así el tercer espacio ofrece una forma distinta de interpretar la geografía humana, más globalizadora y abre la posibilidad de dialogar con otras disciplinas.

Desde finales del siglo XX, las transformaciones económicas, sociales, ambientales y culturales produjeron la visibilización, en la dimensión espacial, del espacio-tiempo. Por tanto, "El desarrollo de las tecnologías de la producción, de la información y la comunicación hizo que se creara simultaneidad temporal entre puntos cada vez más distantes en el espacio" (De Sousa Santos, 2000, p. 220); de forma que se modificaron las prácticas sociales y nuestra experiencia personal. Es así como, John Berger (1987) afirmó que las personas no debían hacer su historia, sino principalmente su geografía; "estamos viviendo una situación histórica que puede ser descrita en términos geográficos" (Citado en 
De Sousa Santos, 2000, p. 220). La forma en que ahora concebimos el espacio va más allá de la discusión filosófica y rejuvenece la teoría social crítica, inspirada en la tradición marxista y en adaptaciones del análisis materialista histórico.

Actualmente, en la Sociedad de la Información y la Comunicación (SIC) tales simultaneidades transforman el espacio respondiendo a los intereses del capital global, el cual intenta estandarizar los lugares, las personas, los gustos, las modas, los patrones de consumo, entre otros. En este contexto, el estudio de la cultura y la trialéctica espacial, desde la perspectiva crítica, en la investigación geográfica, es un tema reciente que interpreta la forma en que las prácticas culturales dejan una impronta en los territorios y los lugares.

El giro espacial, hacia un tercer espacio, plantea la ruptura con las perspectivas tradicionales con las que trabaja la geografía en el primer espacio; de ahí la ausencia o la invisibilización que había de la alteridad en los trabajos geográficos. El constructivismo geográfico ofrece la posibilidad de comprender la espacialidad de una forma distinta de la que hasta ahora se ha trabajado (Lindón, 2012b).

Desde la perspectiva de Benach \& Albet (2010) la hegemonía del espacio percibido (primer espacio), producido y reproducido por prácticas espaciales hegemónicas, otorgó a la geografía un carácter descriptivo; de esta forma el espacio como categoría de análisis central en la geografía fue invisibilizado dentro de las ciencias sociales. Así lo afirma Soja en su libro Postmodern Geographies (1989) durante el siglo XX la geografía evolucionó como una disciplina periférica, aislada, introvertida, de carácter descriptivo, sin profundizar en sus fundamentos teóricos.

Hacia finales del siglo XX e inicio del XXI, el espacio se transforma de forma privilegiada de pensar y actuar, por tanto "las representaciones sociales del espacio adquirirán cada vez mayor importancia y centralidad analíticas" (De Sousa Santos, 2000, p. 220). Tales representaciones serán interpretadas bajo la trialéctica espacial, propuesta por Lefebvre; el espacio percibido alude a las representaciones hegemónicas entendidas como las "prácticas espaciales" que configuran un espacio hegemónico y heterogéneo. El espacio concebido se refiere a las representaciones del espacio, de la semiología y la 
ideología. Por su parte para (Carou \& Bringel, 2010), el espacio vivido es aquel de la "contrarepresentación”, para aludir a las representaciones que desafían el orden dominante.

En este apartado se muestra la dominancia de las representaciones del espacio y la alternativa de las contra-representaciones sociales del espacio en el contexto de la globalización, bajo los planteamiento de la sociología de las ausencias enunciados por De Sousa. La construcción de un tercer espacio se plantea desde la teoría crítica, ya que esta no reduce la realidad de lo existente, sino se imagina "como un campo de posibilidades y la tarea de la teoría define y valora la naturaleza y el ámbito de alternativas a lo que está empíricamente dado" (De Sousa Santos, 2010, p. 23).

\section{Representaciones del primer espacio}

La hegemonía del espacio abstracto y su representación cartográfica tiene lugar en la denominada colonialidad del saber, la que subalterna e invisibiliza saberes no europeos, no blancos, no masculinos, por medio de la dominación epistémica. De esta manera, la representación espacial del espacio abstracto se concreta por medio de una cartografía eurocéntrica; la cual fue inventada por los europeos para destituir las representaciones espaciales y las identidades creadas por los pueblos que habitaban América antes de la llegada de los colonizadores, por tanto, estas representaciones son producciones sociales de la colonia.

El espacio abstracto muestra una imagen única en la representación del mundo, impuesta por la ciencia moderna y expresada en una visión euclidiana y cartesiana. La colonialidad constituye una parte esencial del proceso de consolidación de la hegemonía epistémica y cartográfica de Europa; así la representación única del espacio se impuso como una verdad absoluta sobrepuesta sobre otras formas. La utilidad práctica de esa cartografía respondía a los intereses comerciales de los centros colonizadores interesados en estimar las distancias hacia los lugares colonizados, de forma que deslegitimaban otros raciocinios espaciales. 
Cinco siglos, la cartografía es producida con fines geopolíticos para el uso y control por parte de los estados y anula las posibilidades de existencia de otras representaciones espaciales. La cartografía se construye con fines prácticos, pero a la vez, políticos; distorsionando la realidad sin que la representación sea arbitraria.

El mapa es una imagen de representación del espacio de algo que existe y para esto requiere elementos básicos: la escala, la proyección y la simbología; como representación matemática modelada de la realidad requiere de simplificaciones, lo que (De Sousa Santos, 2000) llama distorsión de la realidad. El primer elemento, la escala de los mapas se clasifica en escala grande y escala pequeña; la primera puede brindar gran cantidad de detalle, aunque de un territorio relativamente pequeño, por ejemplo el barrio, el distrito, el cantón. Al contrario la escala pequeña es poco detallada, pero da información de un territorio mayor: la provincia, el país, la región. El mecanismo de la escala ofrece control sobre el territorio, ya que se pueden diseñar estrategias en pequeña escala, no obstante su accionar se realiza a gran escala; así "la representación/distorsión de la realidad es un presupuesto del ejercicio del poder" (De Sousa, 2000, p. 229).

La proyección, como segundo elemento del mapa, permite la transformación de una superficie curva a una plana, y no puede ocurrir sin crear distorsión en las formas y distancias. Según (Andreis, 2011) “...200 son las formas conocidas y reconocidas de proyecciones cartográficas” (p. 22). El tipo y grado de distorsión es una decisión que privilegia la ideología del cartógrafo y el uso específico que se otorga al mapa. La imposición de la proyección de Mercator, donde exagera las latitudes elevadas y medias en detrimento de las intertropicales, se asocia al proyecto colonialista eurocentrista, además fue utilizado durante la guerra fría para dramatizar la amenaza comunista.

La simbolización, como tercer elemento del mapa, ofrece al lector los códigos simbólicos y convencionales que permiten la interpretación del mapa, donde la semiótica suministra los instrumentos analíticos (De Sousa, 2000). 
La visión euclidiana del espacio representada por una "Cartografía del Poder" inculca "una forma de raciocinio espacial, engendrada en el seno de las relaciones sociales eurocéntricas, que a través de la racionalidad científica, como única forma de raciocinio creíble, promueve a) la opresión epistémica b) la jerarquización del saber c) la uniformización del conocimiento d) la invisibilización de los pueblos, saberes y sus inscripciones territoriales" (Boynard, 2013, p. 6-7).

La colonialidad del saber impuesta por la cartografía eurocéntrica provoca la ausencia (del ignorante), ya que el canon cartesiano y euclidiano es la convención aceptada y medida bajo los criterios de la matemática occidental. Bajo esta concepción única del espacio se excluyen otras formas ancestrales de representación del espacio.

Representaciones del segundo espacio

La representación cartográfica y abstracta ofrece una realidad única dentro del paradigma hegemónico. No obstante, en la vida cotidiana las personas conviven con su espacialidad, la cual les permite tomar decisiones acerca de su accionar diario. Construimos representaciones espaciales que funcionan a manera de mapa mental o cognitivo y éstas desencadenan pensamientos y acciones que determinan nuestro actuar cotidiano.

Otras formas de representaciones espaciales son las “(...) imágenes, diseños, fotografías, descripciones orales, de sueños, mapas mentales, imaginación, etc. Las representaciones espaciales también atraviesan los tiempos en forma de cantos y música” (Boynard, 2013, p.4). A diferencia de las representaciones del espacio abstracto, o el primer espacio, estas involucran los diferentes lenguajes: visual, textual, verbal, gestual, entre otros.

El segundo espacio (espacio concebido), es de orden subjetivo y simbólico cargado de sentimientos, valores y significados. En la dimensión subjetiva, las representaciones de los espacios se crean desde los lugares y las interpretamos por medio de los diferentes sentidos. Así, la capacidad de observación está vinculada fuertemente con nuestras experiencias; para Tuan la perspectiva experiencial, 
entendida como las formas de comprender y organizar la realidad, involucra el uso de los sentidos de la vista, el olfato, el gusto, el tacto para formar una codificación y simbolizar el espacio. La experiencia espacial produce aprendizaje espacial que permite comprender los lugares de los que formamos parte. Estas representaciones involucran sentimientos y valores de las personas respecto al lugar; así que cuando utilizamos los sentidos para ver y oír, no hacemos referencia a lo que observamos u oímos, sino a la forma en que interpretamos o escuchamos. La hermenéutica es una herramienta fundamental.

Estos sentimientos o valores se circunscriben al lugar que está cargado de símbolos y códigos que le otorgan significado, así como valores personales y colectivos. De ahí, que los lugares están cargados de simbologías, signos, relaciones y por tanto representaciones distintas. El apego o desapego al lugar constituye el repositorio de nuestras memorias que nos produce alegría, nostalgia, temor, tristeza, inseguridad, seguridad, sin experimentarlos personalmente. Así, en este contexto es común la valorización o desvalorización de los lugares de lo que existe en comparación con lo demás.

Las representaciones espaciales no constituyen una realidad, "pero consiguen producir sujetos, lo que se entiende por real ya trae en la esencia de esa comprensión una lectura, o sea, lo real es una representación” (Andreis, 2011, p. 25). Algunas expresiones de estas representaciones, tales como mapas mentales o cognitivos, las imágenes, fotografías, el arte, la música producen una ruptura en la monocultura de la clasificación social, ya que tales representaciones son filtradas por la cultura, el género, el grupo social, la edad, entre otras. Esto permite visibilizar las ausencias en la cartografía convencional e imaginar nuestra espacialidad.

\section{Representaciones del tercer espacio}

La comprensión del tercer espacio (espacio vivido) es una forma diferente de pensar las geografías humanas, a la vez, constituye una interpretación crítica del espacio. Aunque la resignificación de la espacialidad toma importancia, con el denominado giro espacial, muchos geógrafos siguen trabajando en el primer y segundo espacio y por tanto la reconceptualización de la espacialidad proviene de fuera de la geografía. Fueron los trabajos de Foucault y Lefebvre, por medio de la imaginación geográfica, 
los que condujeron a una forma distinta de explicar y actuar para apreciar la espacialidad de la vida humana (Benach \& Albet, 2010).

El dualismo entre el enfoque materialista y mental del espacio, lo que Lefebvre (1996) llamó prácticas espaciales y representaciones del espacio, respectivamente, constituye un reduccionismo propio de la teoría social occidental. Tales dualismos confinan la imaginación geográfica y no logran capturar "la complejidad de la experiencia, la amplitud y el tal vez el inescrutable misterio del espacio vivido..." o lo que él llamó Espacios de Representación” (Benach \& Albet, 2010, p. 192).

Lefebvre busca escaparse de la gran dicotomía limitadora al introducir otro término, o una tercera posibilidad, una lógica distinta, más flexible que reconstruya, y a la vez amplíe, la oposición original (Benach \& Albet, 2010); lo que condujo a trascender la dialéctica marxista según el materialismo histórico, para (Clua \& Zusman, 2002) "A la historicidad y al carácter social de las relaciones humanas descritas por Marx (relaciones de producción en un mundo capitalista) cabría agregar la espacialidad" ( 115$)$.

La necesidad de luchar por el derecho a ser diferente en medio de las fuerzas homogeneizadoras, fragmentadoras y la jerarquía del poder de la geografía, en el contexto capitalista, constituye el argumento central de Lefebvre. Las luchas por el derecho a ser diferentes se elaboran en diferentes escalas que van desde el cuerpo, el barrio, la ciudad, la región cultural, los movimientos de liberación nacional, hasta los problemas de desarrollo desigual y subdesarrollo (Soja, 1996. Citado en Clua \& Zusman, 2002).

El tercer espacio (espacio vivido) se convierte en una manera de pensar, representar y actuar para cambiar la espacialidad de la vida; una parte integral de la trialéctica de espacialidad, que puede ser complementaria al primero y segundo espacio. Asimismo, es la perspectiva más globalizadora y ofrece un lugar de encuentro para fomentar la acción política colectiva contra formas de opresión (Benach \& Albet, 2010). 
El reconocimiento más creativo del tercer espacio, en el contexto de la imaginación geográfica, procede de los estudios culturales críticos; fundamentalmente los postcoloniales y los feministas que abordan las temáticas de clase-raza y género. El aporte de estos estudios permite a la geografía humana construir puentes hacia la transdisciplinariedad (Benach \& Albet, 2010).

La conexión entre la geografía y los estudios postcoloniales procuran enriquecer la comprensión de nuevos espacios para la representación del mundo, de una manera distinta. Además posibilita mayor crítica y reflexión alrededor del giro disciplinario para develar los nuevos estudios del desarrollo y aproximaciones críticas a la globalización (Mc Ewan, 2003. Citado Nayak, 2011). Tradicionalmente, la hegemonía del espacio colonial atribuye, a las representaciones espaciales de las antiguas colonias, el simbolismo de las teorías metropolitanas. Para Anthony King (2003) la materialidad, las formas de los edificios, los espacios físicos de la ciudad son elementos que cautivan porque estos reproducen las relaciones, las identidades y subjetividades (Citado en Nayak, 2011).

El abordaje del tercer espacio proviene de los estudios interdisciplinarios de la cultura, así Bell Hooks enriquece la comprensión del espacio vivido al tratar los temas de raza, la clase y género. Sus escritos proporcionan una base política para la luchas colectivas contra la opresión desde diferentes escalas geográfica que van desde el cuerpo hasta la política global (Nayak, 2011).

Con el postmodernismo, el postcolonialismo y el postfeminismo se llama la atención a las sensibilidades que cruzan las fronteras de clase, raza, género, entre otras; se considera "una nueva concepción cultural" (Hernando, 2003, p. 113) que cada vez más se abordan desde otros lugares como: la ciencia, la cultura, el arte. Para Gwendolyn Díaz (2003) nos encontramos ante un nuevo espacio cultural, al cual Hommi Bhabha denomina el Tercer Espacio, un lugar que aparece definido por la diferencia y la otredad. No corresponde a un lugar geográfico, es producto de la presión cultural que filtran las influencias de la cultura dominante y la subordinada (Hernando, 2003). En este sentido, la concepción de Hommi Bhabha y Soja (1996) coinciden en que el tercer espacio propicia el diálogo entre los distintos saberes para avanzar hacia otra forma alternativa de conceptualizar la realidad social. 
Este tercer espacio, a su vez es conocido como "hibridación cultural” y se encuentra en la música, la comida, las bebidas, la moda, el arte, por tanto estos nuevos espacios crean prácticas alternativas y puntos de identificación; así como estrategias en la interpretación simbólica. Más que un acto de complicidad o imitación colonial puede involucrar subversión a la autoridad, puede incitar "nuevas formas de conocimiento, nuevos modos de diferenciación, nuevos sitios de poder (Bhabha, 1994, p. 120. Citado en Nayak, 2011, p. 273).

Por su parte, Gloria Anzaldúa, explica que se requieren teorías que crucen fronteras, donde se reescriba la historia utilizando como categorías la raza, la clase, el género, la etnicidad (citado en Benach \& Albet, 2010). La concurrencia de dos o más corrientes genéricas, "la mezcla de razas, no da por resultado una raza inferior sino que, por el contrario, produce un ser híbrido, una especie donde confluyen fuentes genéticas diversas, lo que produce una cultura ricamente dotada de una multiplicidad de tradiciones" (Hernando, 2003, p. 113).

Finalmente, el tercer espacio o espacio vivido se convierte en un sitio de resistencia, de esas representaciones espaciales contra-hegemónicas, caracterizadas por la contradicción, la opresión; a la vez constituyen espacios de esperanza, de solidaridad que emergen en la sociedad actual, es el otro lugar posible.

\section{REFERÊNCIAS}

Augé, M. (2008). Los no lugares: espacios del anonimato: Gedisa Barcelona.

Andreis, A. M. (2011). La producción de significados y representaciones del espacio por la geografía escolar: posibilidades y limitaciones en los mapas. Revista Geográfica de América Central, 2(45), 15-29.

Benach, N. A., Albert. (2010). Edward W. Soja. La perspectiva postmoderna de un geógrafo radical. Barcelona: Icaria espacios críticos.

Boynard, L. M. (2013). Cartografia e a Colonialidade do Poder. Paper presented at the XIV Encuentro de Geógrafos de América Latina: Reencuentro de saberes territoriales latinoamericanos, Lima, Perú. http://www.egal2013.pe/wpcontent/uploads/2013/07/Tra_Lya-Moret-Boynard.pdf

Carou, H. C., \& Bringel, B. (2010). Articulaciones del Sur Global: afinidad cultural, internacionalismo solidario e Iberoamérica en la globalización contrahegemónica. Geopolítica (s). Revista de estudios sobre espacio y poder, 1(1), 41-63. 
Clua, A., \& Zusman, P. (2002). Más que palabras: otros mundos. Por una geografía cultural crítica. Boletín de la Asociación de Geógrafos Españoles(34), 105-118.

De Sousa Santos, B. (2000). Crítica de la razón indolente, contra el desperdicio de la experiencia. In C. Editora (Ed.), Una cartografía simbólica de las representaciones sociales: el caso del derecho (pp. 223-255). Sao Paulo.

Escobar, A. (2000). El lugar de la naturaleza y la naturaleza del lugar:¿̨ globalización o postdesarrollo? Viola, Andreu comp. Antropología del desarrollo. Teorías y estudios etnográficos en América Latina, 169-216. Retrieved from http://169.158.82.130/cvf/sub-comunidades/gemas/sesion-cientifica-28-05-2013-gemas/El_lugar_de_la_naturaleza.pdf

Hernando, A. M. (2003). El tercer espacio: cruce de culturas en la literatura de frontera. Revista de literaturas modernas, (33-34), 109. Retrieved from http://bdigital.uncu.edu.ar/objetos_digitales/142/Hernando\%20RML34.pdf

Hiernaux-Nicolas, D. (2008). "Geografía objetiva" versus "geografía sensible": trayectorias divergentes de la Geografía Humana en el siglo XX. Revista da ANPEGE, (4), 29-45. Retrieved from http://www.anpege.org.br/revista/ojs2.2.2/index.php/anpege08/article/viewArticle/9

Lefebvre, H., \& Nicholson-Smith, D. (1991). The production of space (Vol. 30): Blackwell Oxford.

Lindón, Aguilar, M., \& Hiernaux, D. (2006). Lugares e imaginarios en la metrópolis. México: Anthropos.

Lindón, A. (2007). Los imaginarios urbanos y el constructivismo geográfico: los hologramas espaciales. Eure (Santiago), 33(99), 31-46. Retrieved from http://www.scielo.cl/pdf/eure/v33n99/art04.pdf

Lindón, A. (2009). La construcción socioespacial de la ciudad: el sujeto cuerpo y el sujeto sentimiento. Revista Latinoamericana de Estudios sobre Cuerpos, Emociones y Sociedad(1), 06-20.

Lindón, A. (2012a). Corporalidades, emociones y espacialidades. Revista Brasileira de Sociologia da Emoção, 11(33), 698 723.

Lindón, A. (2012b). La concurrencia de lo espacial y lo social. In E. Garza \& G. Leyva (Eds.), Tratado de metodología de las ciencias sociales:perspectivas actuales. México: FCE,UAM -Iztapalapa.

Lindón, A., \& Hiernaux, D. (2012). Geografías de lo imaginario. Retrieved from http://www.dhiernauxdocencia.terrageo.net/curso_GeografiaUrbana/lecturas/archivos/5-Hiernaux-2012.pdf

Najmanovich, D. (2011). El juego de los vinculos: subjetividad y redes: figuras en mutación (II ed.). Buenos aires: Biblios.

Nayak, A. J. A. (2011). Geographical thought: an intoduction to ideas in human geography. England: Prentice Hall.

Santos, M. (1990). Por una geografía nueva. Madrid: Espasa-Calpe S.A.

Santos, M. (2000). La naturaleza del espacio. Madrid: Espasa-Calpe, S.A.

Pérez, D. (2013). Sujeto y subjetividad: entre el discurso hegemónico y el discurso alternativo. Una aproximación al estado de la cuestión. Universidad Nacional.

Pozzoli, M. T. (2006). El sujeto de la complejidad. La construcción de un Modelo Teórico Transdisciplinar. Retrieved from http://polis.revues.org/4921

Tuan, Y.-f. (2006). Humanistic Geography-A Personal View. Progress in Geography, 2(001). Retrieved from http://en.cnki.com.cn/Article_en/CJFDTOTAL-DLKJ200602001.htm 
Tuan, Y.-F., \& de Zapata, F. D. (2007). Topofilia: Un estudio de las percepciones, actitudes y valores sobre el entorno: Melusina. 